
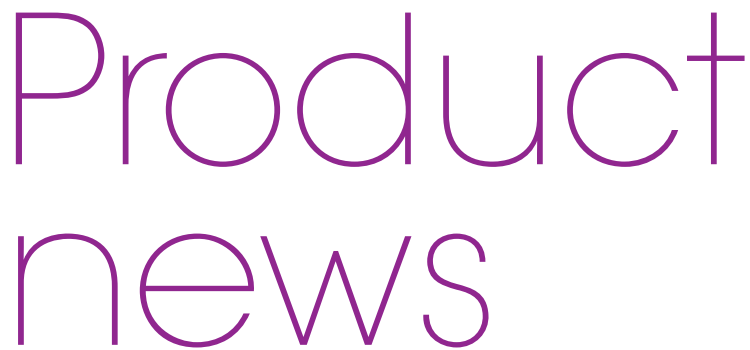

Product news is provided as a service to readers using text and images from the manufacturer, supplier or distributor and does not imply endorsement by BDJ Team. Normal and prudent research should be exercised before purchase or use of any product mentioned.

\title{
AWARD WINNING BABY TOOTHBRUSH
}

Curaprox has won the Best Baby or Kids' Toothbrush category at The Beauty Shortlist Mama \& Baby Awards 2017. Curaprox’s Easy Baby biofunctional toothbrush was developed by dental science specialist Professor Adrian Lussi for use in children of up to four-years-old. One of its many features is the compact, soft rubberised head, which ensures that parents can reach all areas of their child's mouth both effectively and safely, without the danger of inflicting injury to the oral mucosa. The toothbrush has the added benefit of a rounded handle to encourage children at the upper end of the suggested age scale to hold and use the toothbrush correctly, as well as prevent excessive pressure being applied.

In addition, the Easy Baby biofunctional toothbrush employs the use of Curaprox's famous CUREN bristles, which are incredibly fine and gentle on the mouth.

There are a number of other products available within the Curaprox Baby range, all of which have been created in collaboration with orthodontists and specialists to give infants and young children the best possible start in maintaining their oral health. The CURAbaby teething ring, for instance, is available in both pink and blue for infants 0-24 months, and is perfect for reducing pain in teething babies, not to mention ideal for preparing babies for their first toothbrush. Then there's the Curaprox soother, designed to prevent malocclusion and the development of an abnormally high arch through the use of side wings and a flat tip. There are three sizes available: size 0 for 0-7 months, size 1 for 7-18 months and size 2 for 18-36 months. If you'd like to offer your patients the award winning Easy Baby toothbrush or one of the other products available, get in touch with Curaprox today. Call 01480 862084 or visit www. curaprox.co.uk.

\section{ORTHODONTIC AND BRACE CARE}

The common denominator of second teeth and the hormonal changes of adolescence is the depletion of hyaluronic acid, the normal constituent of healthy periodontal tissues. The consequence of this is a reduction in the level of protection given to the mucous membranes of the oral cavity. Gengigel Gel Teen has been formulated to adhere to the mucosa, allowing high molecular weight hyaluronan to penetrate the gums below and restore a healthy balance.

Gengigel is also ideal for children wearing braces, who are more susceptible to gum inflammation, ulcers and lesions caused by rubbing of the appliance. The gel is designed to be massaged gently onto affected areas where it is clinically proven to alleviate pain and rapidly repair damaged gums.

For more information call 02084597550 or email marketing@dentocare.co.uk.

\section{NEW EMERGENCY} EYEWASH STATION

\section{TAUB Products, a long-time}

manufacturer of dental laboratory and restorative products, has introduced a

low-profile emergency eyewash station

that adapts to a wide range of office and laboratory faucets. The new station provides instant access to a single aerated water jet, flushing the eyes and washing the face of chemical or particulate contamination.

The unique design allows water to flow normally downward for washing hands, and during an emergency, the user can quickly rotate it upwards, providing a soft stream of water that will wash the face. The user places the water stream on the brow of the nose and both eyes receive an effective rinse.

Setting this apart from dual-jet designs, the key features of the product include a single rotating nozzle to wash eyes and face, a low-profile modern design that fits most standard faucets, a flow rate of 1.2 gallons per minute, which exceeds ANSI standards, and a design that prevents standing water, thus reducing the chance for mould development.

TAUB Products is celebrating its 65 th year in business. TAUB provides innovative, high-quality solutions for dental professionals. For more information on TAUB and its products go to www.taubdental.com.

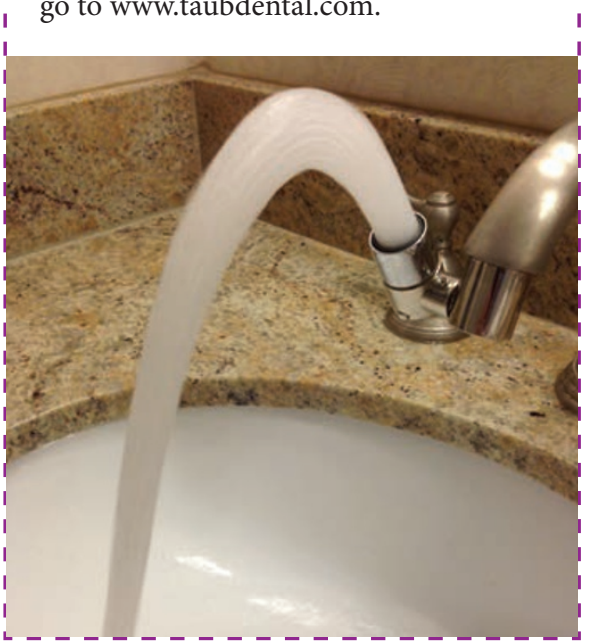

If you would like to promote your products or services direct to the dental industry in BDJ Team, call Andy May on 02078434785 or emaila.may@nature.com. 